\title{
Identificação de Danos Estruturais utilizando o Modelo de Superfície de Resposta e o Método de Monte Carlo com Cadeias de Markov
}

\author{
Isabela Cristina da Silveira e Silva Range ${ }^{1}$ \\ Diego Campos Knupp ${ }^{2}$ \\ Leonardo Tavares Stutz ${ }^{3}$ \\ Luciano dos Santos Rangel ${ }^{4}$ \\ Programa de Pós-Graduação em Modelagem Computacional, Instituto Politécnico, Universidade \\ do Estado do Rio de Janeiro, Nova Friburgo, RJ
}

\begin{abstract}
Resumo. O presente trabalho aborda o problema de identificação de danos em vigas a partir de um Modelo de Superfície de Resposta (MSR) da matriz de flexibilidade estrutural . O dano é descrito por um parâmetro de coesão, discretizado espacialmente pelo Método dos Elementos Finitos (MEF). O problema inverso é formulado segundo a abordagem Bayesiana, de modo que a solução do problema consiste na determinação de densidades de probabilidade marginais a posteriori dos parâmetros nodais de coesão. O método de Monte Carlo com Cadeias de Markov (MCMC) foi utilizado para a obtenção das densidades de probabilidade. Diante dos resultados obtidos, pode-se concluir que a abordagem proposta foi capaz de identificar com acurácia os campos de dano considerados.
\end{abstract}

Palavras-chave. Identificação de Danos, Matriz de Flexibilidade, Superfície de Resposta, Inferência Bayesiana, Cadeias de Markov.

\section{Introdução}

Nos últimos anos, o problema de identificação de danos estruturais tem despertado o interesse de vários pesquisadores, possibilitando a redução dos custos operacionais [2]. Os métodos de identificação de danos, fundamentados na resposta dinâmica da estrutura, vem se tornando uma prática utilizada nas indústrias da construção civil, mecânica e aeroespacial. Sendo classificados, de forma geral, em três tipos, de acordo com o domínio dos dados utilizados: domínio do tempo, domínio da frequência e nas formas modais [5].

Neste trabalho, a identificação de danos estruturais é realizada utilizando os dados modais da estrutura, especificamente a matriz de flexibilidade. Geralmente as técnicas de identificação de danos estruturais são fundamentadas no ajuste de um Modelo de Elementos Finitos (MEF). Outra técnica, recentemente proposta, de identificação de danos

\footnotetext{
${ }^{1}$ isilva@iprj.uerj.br

${ }^{2}$ diegoknupp@iprj.uerj.br

${ }^{3}$ ltstutz@iprj.uerj.br

${ }^{4}$ lrangel@iprj.uerj.br
} 
é baseada no Modelo de Superfície de Resposta (MSR), que está ganhando popularidade devido à sua eficiência computacional [4]. Portanto, na formulação do problema inverso de identificação de danos, considerou-se o ajuste de um MSR, em substituição do MEF, de uma viga de Euler-Bernoulli simplesmente apoiada.

O problema inverso de identificação de danos é realizado a partir da inferência Bayesiana. Dentre suas vantagens, pode-se destacar a capacidade de incluir informações a priori, a facilidade de incorporá-las em um contexto formal de decisão, o tratamento explícito das incertezas do problema e a habilidade de assimilar novas informações em contextos adaptativos [8]. A estimação de parâmetros é obtida com o método de Monte Carlo com Cadeias de Markov (MCMC), usando o algoritmo Metropolis-Hasting com uma distribuição priori gaussiana.

\section{Modelagem Matemática do Problema Direto}

$\mathrm{Na}$ abordagem adotada no presente trabalho, o dano é continuamente descrito ao longo da estrutura por um parâmetro de coesão definido como

$$
\beta(x)=\frac{E(x) I(x)}{E_{0} I_{0}},
$$

onde $E(x)$ e $I(x)$ são, respectivamente, o módulo de elasticidade e o momento de inércia de área da seção transversal e $E_{0}$ e $I_{0}$ são os correspondentes valores nominais. Esse parâmetro relaciona-se com a ligação entre os pontos materiais e pode ser interpretado como uma medida do estado de coesão local do material, onde $0 \leq \beta \leq 1$ [6].

Considerando, por simplicidade, que a viga possui seção retangular e que o módulo de elasticidade é uniforme, de acordo com a Eq. (1), tem-se

$$
\beta(x)=\left(\frac{h(x)}{h_{0}}\right)^{3},
$$

onde $h_{0}$ e $h(x)$, indicam, respectivamente, a espessura nominal e a espessura da viga na posição $x$. Discretizando espacialmente o campo de coesão em $n_{p}$ nós, pelo MEF, tem-se o vetor de parâmetros nodais de coesão

$$
\boldsymbol{\beta}=\left[\beta_{1}, \beta_{2}, \ldots, \beta_{n_{p}}\right]^{T} .
$$

A matriz de flexibilidade da estrutura pode ser escrita como

$$
\mathbf{G}=\sum_{i=1}^{n} \frac{1}{\omega_{i}^{2}} \phi_{i} \phi_{i}^{T},
$$

onde $n$ representa o número de graus de liberdade (GDL) do MEF do sistema, $\omega_{i}$ é a i-ésima frequência natural não-amortecida e $\phi_{i}$ é a i-ésima forma modal da estrutura [7], normalizada em relação à matriz de massa, obtidas do problema de autovalor generalizado

$$
\left(\mathbf{K}-\omega_{i}^{2} \mathbf{M}\right) \phi_{i}=0, \quad i=1,2, \ldots, n .
$$


Como se pode observar a partir da Eq. (4), devido à relação inversa com o valor quadrático das frequências naturais, pode-se obter uma estimativa precisa da matriz de flexibilidade a partir dos modos de mais baixa frequência da estrutura. Dado que os modos de frequência mais elevada são mais difíceis de serem obtidos em um ensaio experimental, a rápida convergência da matriz de flexibilidade representa uma grande vantagem para a sua utilização na formulação do problema inverso de identificação de danos. Devido às limitações práticas inerentes aos testes modais, a seguinte aproximação da matriz de flexibilidade pode ser obtida experimentalmente

$$
\mathbf{G}_{E}=\sum_{i=1}^{n_{E}} \frac{1}{\omega_{i E}^{2}} \phi_{i E} \boldsymbol{\phi}_{i E}^{T}
$$

onde $n_{E}<n$ é o número de modos obtidos do ensaio experimental, $\omega_{i E}$ e $\phi_{i E}$ são, respectivamente, a i-ésima frequência natural não-amortecida e a forma modal obtidas experimentalmente. Note-se que a ordem da matriz de flexibilidade experimental $\mathbf{G}_{E}$ depende apenas do número $m$ de GDL medidos no ensaio modal, que é equivalente ao número de componentes das formas modais experimentais $\phi_{i E}$.

\section{Modelo de Superfície de Resposta}

Em um Modelo de Superfície de Resposta (MSR), relações explícitas são definidas entre os parâmetros da estrutura e a resposta de interesse. No caso, entre os parâmetros nodais de coesão e os elementos da matriz de flexibilidade estrutural. Assim, para uma dada resposta escalar $g$, tem-se

$$
g=f\left(\beta_{1}, \beta_{2}, \ldots, \beta_{n_{p}}\right)+\varepsilon,
$$

onde $f$ é uma superfície de resposta, ou função de resposta, e $\varepsilon$ representa o erro de predição do modelo, que é considerado como uma variável aleatória normalmente distribuída com média nula. Em geral, os parâmetros do modelo são codificados como

$$
x_{i}=\frac{\beta_{i}-\left(\beta_{\min }+\beta_{\max }\right) / 2}{\left(\beta_{\max }-\beta_{\min }\right) / 2}, \quad i=1,2, \ldots, n_{p},
$$

tal que $x_{i} \in[-1,1] ; \beta_{\min }$ e $\beta_{\max }$ são, respectivamente, os valores mínimo e máximo dos parâmetros de coesão considerados na determinação do MSR.

Nesse trabalho, será considerada uma superfície de resposta polinomial de segunda ordem, dada por

$$
\hat{g}=f\left(x_{1}, x_{2}, \ldots, x_{n_{p}}\right)=\hat{\alpha}_{0}+\sum_{i=1}^{n_{p}} \hat{\alpha}_{i} x_{i}+\sum_{i=1}^{n_{p}} \hat{\alpha}_{i i} x_{i}^{2}+\sum_{i<j}^{n_{p}} \sum_{j=2}^{n_{p}} \hat{\alpha}_{i j} x_{i} x_{j} .
$$

onde $\hat{\alpha}_{i}, \hat{\alpha}_{i i}$ e $\hat{\alpha_{i j}}$ são os coeficientes da superfície de resposta, estimados pelo método dos mínimos quadrados [5]. 
De acordo com a Eq. (9), o número de coeficientes do polinômio adotado é $n_{c}=\left(n_{p}+\right.$ 1) $\left(n_{p}+2\right) / 2$. Portanto, para a definição apropriada da superfície de resposta, deve-se ter um número de dados maior ou igual ao número de seus coeficientes, $n_{d} \geq n_{c}$. No presente caso, um dado significa o par $(x ; \hat{g})$, isto é, um vector constituído por valores codificados dos parâmetros de coesão, que descreve um cenário de dano, e a correspondente resposta estrutural de interesse. O Projeto Fatorial Fracionado $\left(2^{n_{p}-f}\right)$ será aqui considerado para a escolha dos pontos utilizados na determinação da superfície de resposta utilizada no presente trabalho.

\section{Problema Inverso de Identificação de Danos}

Na Inferência Bayesiana, os parâmetros do modelo são considerados variáveis aleatórias e o objetivo reside na determinação da densidade de probabilidade a posteriori associada a cada parâmetro. Pelo teorema de Bayes, a densidade de probabilidade conjunta a posteriori dos parâmetros é dada por

$$
P_{\text {post }}\left(\boldsymbol{\beta} \mid \boldsymbol{G}_{E}\right)=\frac{P\left(\boldsymbol{G}_{E} \mid \boldsymbol{\beta}\right) P_{p r}(\boldsymbol{\beta})}{P\left(\boldsymbol{G}_{E}\right)}
$$

onde $P\left(\boldsymbol{G}_{E} \mid \boldsymbol{\beta}\right)$, é a função de verossimilhança, $P_{p r}(\boldsymbol{\beta})$ é a densidade de probabilidade $a$ priori e $P\left(\boldsymbol{G}_{E}\right)$ é a densidade de probabilidade das respostas medidas, que desempenha o papel de uma constante de normalização [3].

Considerando-se que os erros experimentais apresentam uma distribuição de probabilidade normal, a verossimilhança é dada por

$$
P\left(\boldsymbol{G}_{E} \mid \boldsymbol{\beta}\right)=\frac{1}{\sqrt{\operatorname{det} \boldsymbol{V}}} \frac{1}{\sqrt{(2 \pi)^{n_{e}}}} \exp \left[-\frac{1}{2}\left(\boldsymbol{G}_{E}-\hat{\boldsymbol{G}}\right)^{T} \boldsymbol{V}^{-1}\left(\boldsymbol{G}_{E}-\hat{\boldsymbol{G}}\right)\right],
$$

sendo $n_{e}$ o número de dados experimentais utilizados no problema, $\boldsymbol{V}$ a variância dos erros experimentais e $\hat{\boldsymbol{G}}$ a resposta prevista pelo MSR.

Para a obtenção da densidade de probabilidade marginal de cada parâmetro, será aqui considerando o método de amostragem de Monte Carlo com Cadeias de Markov (MCMC) [1]. Na geração das cadeias de Markov foi utilizado o algoritmo de MetropolisHastings, que faz uso de uma densidade de probabilidade auxiliar $\mathbf{q}\left(\boldsymbol{\beta}^{*} \mid \boldsymbol{\beta}_{i-1}\right)$, que fornece uma regra para a geração de um vetor candidato $\boldsymbol{\beta}^{*}$, dado um estado atual $\boldsymbol{\beta}_{i-1}$ da cadeia. Sendo o vetor candidato $\boldsymbol{\beta}^{*}$ aceito ou não, de acordo com a probabilidade dada por:

$$
r=\min \left[1, \frac{P\left(\boldsymbol{\beta}^{*}\right) P\left(\boldsymbol{\beta}^{*} \mid \boldsymbol{\beta}_{i-1}\right)}{P\left(\boldsymbol{\beta}_{i-1}\right) P\left(\boldsymbol{\beta}_{i-1} \mid \boldsymbol{\beta}^{*}\right)}\right] .
$$

Se $U \leq r$, onde $U$ é um número aleatório gerado de uma distribuição uniforme entre 0 e 1, então o candidato $\boldsymbol{\beta}^{*}$ é aceito, caso contrário, é rejeitado.

Esse procedimento é repetido $N$ vezes, onde $N$ é o tamanho da cadeia, resultando na cadeia de Markov $\left\{\boldsymbol{\beta}_{1}, \boldsymbol{\beta}_{2}, \cdots, \boldsymbol{\beta}_{N}\right\}$. Os estados gerados até que se alcance o equilíbrio são chamados de amostras de aquecimento (burn-in) e são descartados para a determinação das propriedades estatísticas dos parâmetros de coesão [8]. 


\section{$5 \quad$ Resultados Numéricos}

A metodologia proposta é validada em uma viga de alumínio simplesmente apoiada cujos parâmetros geométricos e materiais são: $1 \mathrm{~m}$ de comprimento, 0, $005 \mathrm{~m}$ de espessura, 0, $05 \mathrm{~m}$ de largura, $2700 \mathrm{~kg} / \mathrm{m}^{3}$ de massa específica, $5,21 \times 10^{-10} \mathrm{~m}^{4}$ e $7,26 \times 10^{10} \mathrm{~Pa}$ de momento de inércia de área e módulo de elasticidade, respectivamente. A viga foi discretizada em 20 elementos bidimensionais do tipo Euler-Bernoulli, onde cada elemento possui dois GDL, totalizando assim, 40 GDL, e um parâmetro de coesão, logo, a viga possui 21 parâmetros nodais de coesão. Considerou-se que apenas os 10 primeiros modos de vibrações foram medidos em 10 GDL da estrutura.

Neste trabalho, o MEF, com os valores dos parâmetros de coesão prescritos de acordo com os cenários de dano foi considerado, para gerar a matriz de flexibilidade experimental sintética, da estrutura danificada. No entanto, no processo de identificação de danos, como modelo direto é utilizado o MSR.

A Tabela 1, apresenta os cenários de dano considerados neste trabalho, onde o nível de ruído representa o percentual de ruído aditivo, na forma modal, para a geração da matriz de flexibilidade experimental.

Tabela 1: Cenários de danos.

\begin{tabular}{|c|c|c|c|c|}
\hline Cenário & Posição $(\mathrm{m})$ & $h(x) / h_{0}$ & $\beta(x)$ & Nível de ruído (\%) \\
\hline 1 & $0,35\left(\beta_{8}\right)$ & 0,8 & 0,512 & 1 \\
\hline 2 & $0,2\left(\beta_{5}\right) ; 0,55\left(\beta_{12}\right)$ & 0,$8 ; 0,8$ & 0,$512 ; 0,512$ & 1 \\
\hline
\end{tabular}

Para todas as simulações foram consideradas cadeias de Markov com 100.000 estados com aquecimento de 80.000 estados. Foi adotada uma distribuição auxiliar uniforme truncada com desvio padrão $\sigma=0,005$ e uma distribuição priori gaussiana com desvio padrão de 0,5 para todos os Casos.

Na Figura 1(a) é apresentado o resultado da identificação de danos, em termo da altura relativa, onde mostra os campos de dano exato e estimado para o Caso 1, além do intervalo de confiança (IC) de 95\%. E, a Figura 1(b) mostra a convergência da cadeia de Markov para o parâmetro danificado $\beta_{8}$.

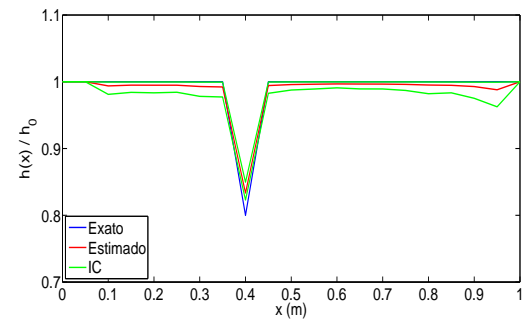

(a) Identificação

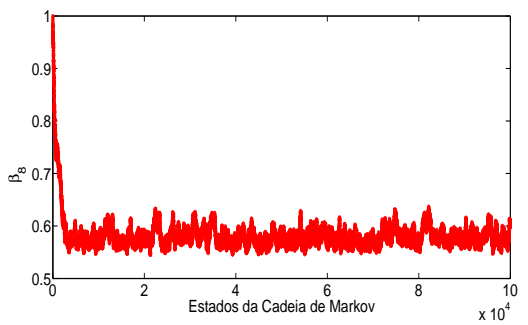

(b) Convergência da Cadeia

Figura 1: Resultados da identificação para o Caso 1. 
Pode-se observar que o MCMC foi capaz de identificar com acurácia a intensidade do dano existente na estrutura, mesmo considerando $1 \%$ de ruído nos modos de vibração. As Figuras 2(a) e 2(b) apresentam o resultado da identificação de danos e a convergência das cadeias de Markov, para os parâmetros danificados $\beta_{5}$ e $\beta_{12}$, do Caso 2.

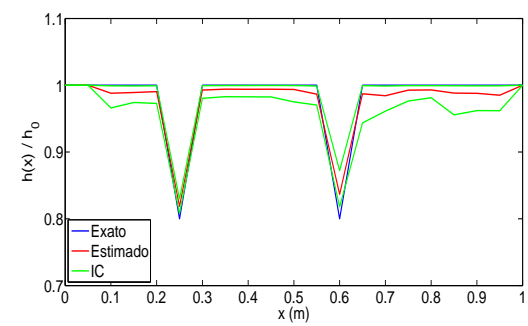

(a) Identificação
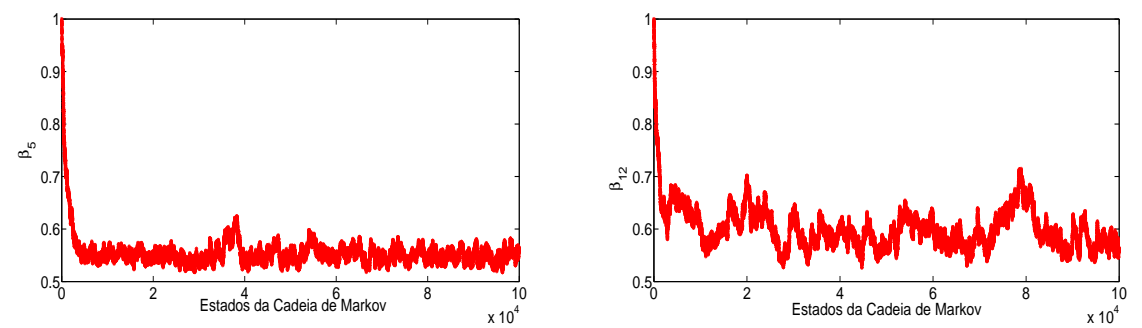

(b) Convergência das Cadeias

Figura 2: Resultados da identificação para o Caso 2.

Com o resultado da identificação de danos apresentado pela Figura 2 pode-se observar que o MCMC identificou as duas posições de dano do Caso 2. Portanto, a partir dos resultados obtidos percebe-se que a estratégia adotada identificou de forma bastante satisfatória os cenários de dano considerados.

\section{Conclusões}

Neste trabalho, o problema de identificação de danos estruturais em uma viga de Euler-Bernoulli simplesmente apoiada foi resolvido a partir de uma abordagem Bayesiana. Incialmente foi ajustado um MSR da estrutura, a fim de substituir o MEF no problema inverso. A solução do problema inverso de estimação, foi obtida pelo método MCMC, implementado através do algoritmo de Metropolis-Hastings. Pelos resultados obtidos, concluiu-se que a estratégia adotada foi capaz de identificar os cenários de danos mesmo considerando a presença de ruído. Como sugestões de trabalhos futuros destaca-se a aplicação da abordagem Bayesiana no problema de identificação de danos em estruturas do tipo placa. 


\section{Agradecimentos}

Os autores deixam aqui registrados os agradecimentos às agências de fomento CAPES, CNPq e FAPERJ pelo apoio concedido.

\section{Referências}

[1] D. C. S. Cuevas. Estimação de Parâmetros de Materiais Heterogêneos com o Método de Monte Carlo com Cadeias de Markov. Dissertação de Mestrado em Engenharia Mecânica, Universidade Federal do Rio de Janeiro, Rio de Janeiro, pages 109, 2014.

[2] S. E. Fang, W. X. Ren and R. Perera. A stochastic model updating method for parameter variability quantification based on response surface models and Monte Carlo simulation. Mechanical Systemsand Signal Processing, 33: 83-96, 2012.

[3] J. P. Kaipio, E. Somersalo. Statistical and Computational Inverse Problems, SpringVerlag, New York, 2004.

[4] T. Mukhopadhyay, T. K. Dey, R. Chowdhury and A. Chakrabarti. Structural Damage Identification Using Response Surface-Based Multi-objective Optimization: A Comparative Study. Arab J. Sci. Eng., 40: 1027-1044, 2015.

[5] I. C. S. S. Rangel, L. S. Rangel e L. T. Stutz. Identificação de Danos Estruturais a partir de um Modelo de Superfície de Resposta. Tendências em Matemática Aplicada e Computacional, 17: 267-282, 2016.

[6] L. T. Stutz, D. A. Castello and F. A. Rochinha, A flexibility-based continuum damage identification approach. Journal of Sound and Vibration, 279: 641-667, 2005.

[7] L. T. Stutz, R. A. Tenenbaum and R. A. P. Correa. The Differential Evolution method applied to continuum damage identification via flexibility matrix. Journal of Sound and Vibration, 345: 86-102, 2015

[8] J. S. Teixeira, L. T. Stutz, D.C. Knupp and A. J. Silva Neto. Application of a Continuum Damage Identification Approach via Markov Chain Monte Carlo Method using the Impulsive Response Function. 8th International Conference on Inverse Problems in Engineering, Cracow, Poland, 2014. 